\title{
Joint Mobility and Routing for Lifetime Elongation in Wireless Sensor Networks
}

\author{
Jun Luo \\ Jean-Pierre Hubaux \\ School of Computer and Communication Sciences \\ Ecole Polytechnique Fédérale de Lausanne (EPFL), CH-1015 Lausanne, Switzerland \\ Email: \{jun.luo, jean-pierre.hubaux\}@epfl.ch
}

\begin{abstract}
Although many energy efficient/conserving routing protocols have been proposed for wireless sensor networks, the concentration of data traffic towards a small number of base stations remains a major threat to the network lifetime. The main reason is that the sensor nodes located near a base station have to relay data for a large part of the network and thus deplete their batteries very quickly. The solution we propose in this paper suggests that the base station be mobile; in this way, the nodes located close to it change over time. Data collection protocols can then be optimized by taking both base station mobility and multi-hop routing into account. We first study the former, and conclude that the best mobility strategy consists in following the periphery of the network (we assume that the sensors are deployed within a circle). We then consider jointly mobility and routing algorithms in this case, and show that a better routing strategy uses a combination of round routes and short paths. We provide a detailed analytical model for each of our statements, and corroborate it with simulation results. We show that the obtained improvement in terms of network lifetime is in the order of $500 \%$.
\end{abstract}

Index Terms-Sensor networks, Network lifetime, Mobile Base Stations, System design, Mathematical optimization, Simulations.

\section{INTRODUCTION}

Many communication protocols for energy conservation in wireless sensor networks have been proposed recently. These include, among others, energy conserving routing (e.g., [1], [2], [3], [4]), topology control (e.g., [5], [6], [7], [8]) and clustering (e.g., [9], [10], [11], [12]). Although all these protocols achieve their optimization goals under certain conditions, they always focus on the sensor nodes. ${ }^{1}$ We will show that further improvements on the lifetime of sensor networks can be achieved if we shift our focus to the behavior of base stations.

We observe that, as data traffic must be concentrated towards a small number (typically one) of base stations, the sensor nodes around a base station have to forward data for other nodes whose number can be very large; this problem always exists, regardless of what energy conserving protocol is used for data transmission. In other words, applying energy

\footnotetext{
${ }^{\dagger}$ The work presented in this paper was supported (in part) by the National Competence Center in Research on Mobile Information and Communication Systems (NCCR-MICS), a center supported by the Swiss National Science Foundation under grant number 5005-67322. (http: //www.terminodes.org)

${ }^{1}$ In this paper, the words sensor, sensor node and node are used interchangeably.
}

conserving protocols does not directly lead to load balancing in the whole network. Using a simple analytical model, we will show how unevenly the load is distributed within a network. As a result, those bottleneck nodes around base stations deplete their batteries much faster than other nodes and, therefore, their lifetime upper bounds the lifetime of the whole network.

Intuitively speaking, the load of sensor nodes can be more balanced if a base station changes its position from time to time. Although a base station is usually assumed to be static, it can become mobile thanks to the advance made in the field of robotics [13], [14]. In this paper, taking a mobile base station into account, we investigate the problem of loadbalanced data collection in wireless sensor networks. The idea is to make use of existing multi-hop routing protocols and to achieve further improvements in terms of network lifetime by exploiting the base station mobility. Since multi-hop routing is used, our solution does not significantly affect latency and is thus different from the mobile relay approach [15], [16]. Using our analytical model, we first show that the load distribution becomes much more even, with only an arbitrary mobility trajectory of the base station. These analytical results suggest that, in addition to routing, mobility (of base stations) does help to optimize the network lifetime. Considering jointly the mobility and routing strategies, we propose data collection protocols that further balance the load. Finally, we perform simulations to validate the analytical model and to quantify the lifetime elongation compared with sensor networks with a static base station.

The rest of this paper is organized as follows: Section II states the problem and the network model. Section III analyzes sensor networks with a static base station and with a base station moving arbitrarily. Section IV refines the problem definition, presents our joint mobility and routing strategy for data collection, and discusses implementation issues. Simulation results are provided in Section V. Section VI surveys related work. Finally, Section VII concludes the paper.

\section{Network Model and Problem Statement}

We assume a relatively dense and strongly connected network that harvests data from the area covered by the network. The network consists of a set $N$ of static sensor nodes and one base station that collects data from all nodes. We focus only on the communications between the nodes and the base station, 
whereas the communications between the base station and devices outside the network are out of the scope of this paper. We also assume that nodes are distributed as a Poisson process with density $\rho$ within a circle $\mathcal{C}_{O R}$ of center $O$ and radius $R$. Each node sends data to the base station with a constant rate $\lambda$. The overall energy for a node to receive and transmit a unit of data is $\varepsilon$. The transmission and sensing ranges of all nodes are identical and fixed at $r(r \ll R)$. For simplicity, we assume an ideal load-balanced short path routing protocol (we refer to [4] for a profile of such protocols), and we do not consider data aggregation (e.g., [17], [18]) when data are collected ${ }^{2}$. Fig. 1 illustrates this model. The model can be extended to cases where multiple base stations exist by dividing the network into several sections with one base station assigned to each.

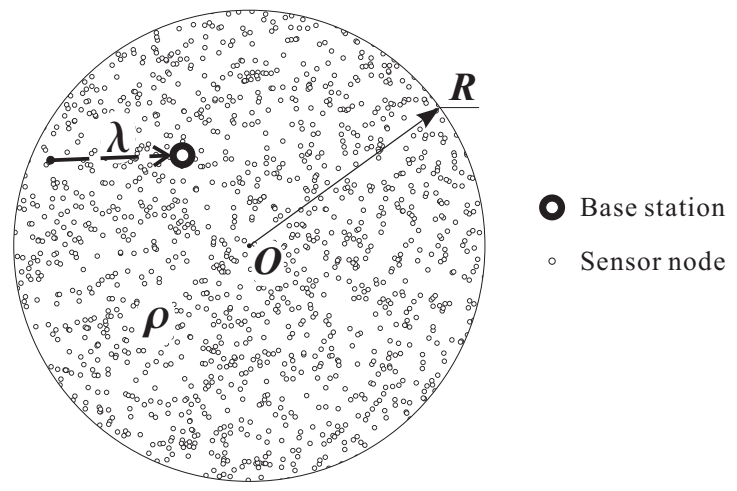

Fig. 1. Network model.

Let us first define the relevant concepts before specifying our problem. We define the network lifetime as the time span from the sensor deployment to the first loss of coverage [20] (i.e., the time when some area initially covered by the network is not sensed by any active node any more). The load of node $n$, $\operatorname{load}_{n}$, is the power that $n$ consumes to transmit and receive data. It is obvious that the higher $\operatorname{load}_{n}$ is, the shorter the lifetime of $n$ is. The average load of $n, \overline{l o a d}_{n}$, is an average over both a time period and a subset of nodes that are within the sensing range of $n$. The time average is necessary if the base station mobility (mobility hereafter) is introduced, since $\operatorname{load}_{n}$ becomes time-variant. And the geographical average makes sense due to our definition of network lifetime; whereas the quick loss of individual nodes bearing a high load may not lead to the loss of coverage, a subset of nodes with a high average load do leave a coverage "hole" in the network after depleting their batteries all together. We also provide our definition of energy efficiency in order to clarify its relationship with the network lifetime: A protocol is energy efficient if it minimizes the accumulative energy consumption for fulfilling its task (e.g., minimum-energy broadcast [21]). It has long

\footnotetext{
${ }^{2}$ On one hand, it can be easily seen that applying these strategies can only improve the performance of our protocols. On the other hand, assuming redundancy in order to use data aggregation becomes impractical if the sensed areas are much smaller than the overall areas under monitoring (for instance, collecting soil moisture over large agricultural areas [19]).
}

been recognized that an energy efficient protocol does not necessarily maximize the network lifetime [1].

Although the network lifetime definition based on the concept of loss of coverage is more comprehensive than other definitions [22], the lifetime defined as such is hard to quantify; existing proposals have investigated only its upper bound [20], [23], [24]. Therefore, we convert the problem of maximizing network lifetime to a problem of load balancing, and formulate it as a min-max problem in terms of the average load of a certain node $n$ :

$$
\begin{aligned}
& \text { Minimize } \quad \operatorname{load}_{N} \equiv \max _{\forall n \in N} \overline{\operatorname{load}}_{n} \text { (strategies) } \\
& \text { Constraints : specific to given strategies. }
\end{aligned}
$$

The intuition behind this formulation is that the network lifetime is roughly in inverse proportion to $\operatorname{load}_{N}$, or the network load. Existing solutions, when specifying the problem decision variables (or strategies) and constraints, only take routing strategies into account. In this paper, we intend to show that, by considering jointly mobility and routing strategies, one can yield a better solution to the problem. But before doing this, we first demonstrate in the next section that mobility is indeed a strategy that deserves to be considered. Note that, in our proposal, the base station does not rely only on mobility to retrieve data from sensors (in opposition to the proposals in [15], [16]); the data collection procedure continues through multi-hop routing wherever the base station stays.

\section{To Move or Not To Move}

In this section, we compare two cases. In one case, we locate the base station in a place where the most energy efficient data collection is achieved. In the other case, we require the base station to move in an arbitrary way. We analytically quantify the benefit in terms of network lifetime due to mobility, and thus show that mobility deserves to be a strategy for solving the problem specified by (1).

\section{A. Networks with a Static Base Station}

We first place the base station at its optimum location in terms of energy efficiency, then we show that the network lifetime is quite limited with this optimum base station position.

Claim 1: The center of the circle $\mathcal{C}_{O R}$ is the optimum position for a base station in terms of energy efficient data collection.

Proof: Let the base station be at $B\left(x_{B}, y_{B}\right)$ and consider an infinitesimal area $S$ that measures $\mathrm{d} x \times \mathrm{d} y$ and is centered on $(x, y)$, as shown in Fig. 2. Given the Euclidean distance $d=\sqrt{\left(x-x_{B}\right)^{2}+\left(y-y_{B}\right)^{2}}$ from the center of $S$ to $B$, the routing path length (in hops) $l$ from $S$ to $B$ is approximately linear in $d$ (i.e., $l \approx k d$ ) due to the assumption of a short path routing protocol. As a result, the energy consumed to transmit data from $S$ to $B$ is $k d \varepsilon \times \lambda t \rho \times \mathrm{d} x \mathrm{~d} y$, where $\lambda t \rho \times \mathrm{d} x \mathrm{~d} y$ is the amount of data produced within time $t$ and $k d \varepsilon$ is the energy spent to transmit a unit of data from $S$ to $B$. The total 


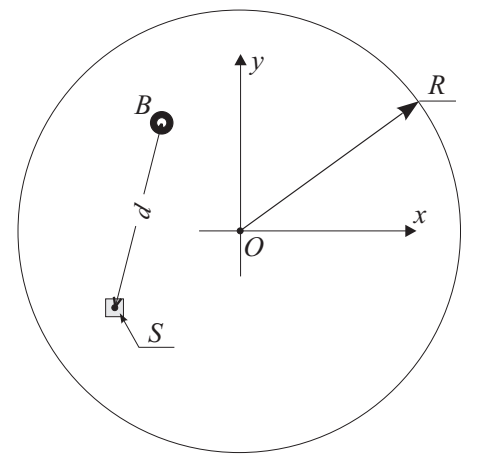

Fig. 2. Proof of optimum base station position.

energy consumption is $E=\int_{y} \int_{x} k d \varepsilon \lambda t \rho \mathrm{d} x \mathrm{~d} y$. It is easy to see that minimizing $E$ is equivalent to minimizing

$$
\begin{array}{r}
\int_{-R}^{+R} \int_{-\sqrt{R^{2}-y^{2}}}^{+\sqrt{R^{2}-y^{2}}}\left[\left(x-x_{B}\right)^{2}+\left(y-y_{B}\right)^{2}\right] \mathrm{d} x \mathrm{~d} y \\
=\frac{1}{2} \pi R^{2}\left(2 x_{B}^{2}+2 y_{B}^{2}+R^{2}\right)
\end{array}
$$

which achieves the minimum value if $x_{B}=y_{B}=0$, i.e., the based station is placed at the center $O$.

We will show that, even with this optimum position, the load is poorly distributed among the nodes. Given a node $n$ that is at distance $d$ from the base station $B$ (also the center $O$ ), as shown Fig. 3, the geographical average load taken by this node is in proportion to $\left(S_{1}+S_{2}\right) / S_{2}$. Here we adopt and extend the model proposed in [25]. In Appendix I, we give detailed explanations about how this model is built. The

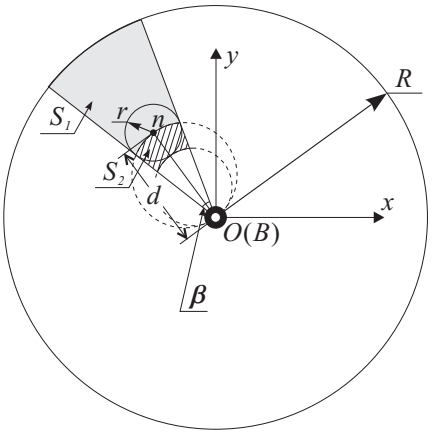

(a) $d \geq r$

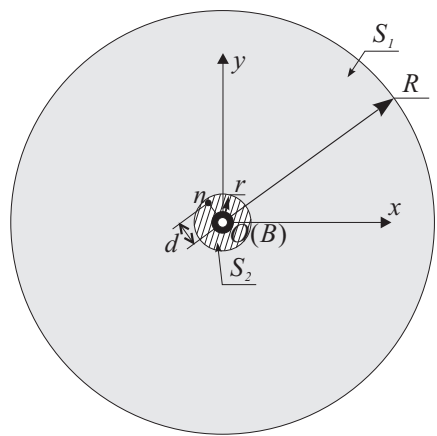

(b) $d<r$
Fig. 3. Calculation of load distribution with a centered static base station. $S_{1}$ and $S_{2}$ in (a) are two disjoint parts of a sector whose two sides are tangent to a circle of center $n$ and radius $r$.

intuition is that all the traffic flows from both areas $S_{1}$ and $S_{2}$ have to go through nodes in area $S_{2}$, which forms a "pressure" on those nodes. The (geographical) average power that a node in $S_{2}$ spends to forward the data traffic can be calculated as the intensity of the pressure:

$$
\begin{aligned}
\overline{\operatorname{load}}_{n}= & \frac{\left(S_{1}+S_{2}\right) \rho \lambda \varepsilon}{S_{2} \rho} \\
& \begin{cases}\approx \frac{\frac{\beta}{2}\left(R^{2}-d^{2}\right) \lambda \varepsilon}{\frac{\pi}{2} r^{2}}+\lambda \varepsilon & d \geq r \\
=\frac{R^{2} \lambda \varepsilon}{r^{2}} & d<r\end{cases}
\end{aligned}
$$

where $\beta=2 \arcsin (r / d)$. As shown in Fig. 4, the average load of a sensor node increases dramatically with the decreasing distance between the node and the base station. This means
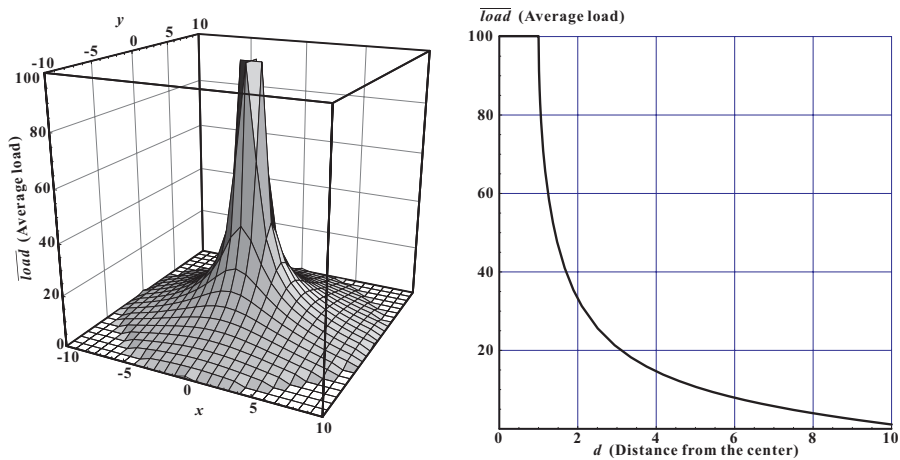

Fig. 4. Load distribution with a centered static base station. We assume $R=10, r=1, \rho=8 / \pi, \lambda=1$, and $\varepsilon=1$.

that the nodes around the base station use up their energy much faster than other nodes, because they have to forward a great amount of traffic flows even though their number is limited. Therefore, the network lifetime is upper bounded by the lifetime of these nodes. Also, when these nodes run out of batteries, the base station has no way to collect data any more (even though a large part of the network is still "alive"), because the network is partitioned. Finally, the $\operatorname{load}_{N}$ does not vary with different base station position, according to (2) with $d<r$. However, the centered position is optimum in terms of energy efficiency, as shown by Claim 1 .

\section{B. Networks with a Moving Base Station}

Intuitively speaking, a moving base station can distribute over time the role of "hot spots" (i.e., the nodes around the base station), such that the load can be evened out. In this section, we prove that this intuition is indeed correct. Since the data collection procedure continues wherever the base station stays, (i) any departure of the base station from the center increases the worst-case latency (whose maximum value doubles compared with that in the case of centered static base station) and (ii) the moving speed is not essential to a mobility strategy.

We assume that the base station moves in such a way that it appears everywhere with the same frequency in the long run. We could continue using the model in Section III-A, but it would result in an extremely complex integral, which can only be computed through numerical methods and does not provide enough insight into the system performance. Therefore, we simplify the model in order to obtain a closed form expression. 
Let us consider the power consumption of an arbitrary node $n$ that is at distance $d$ from the center, with respect to a random base station position $B$. As shown in Fig. 5, we consider that

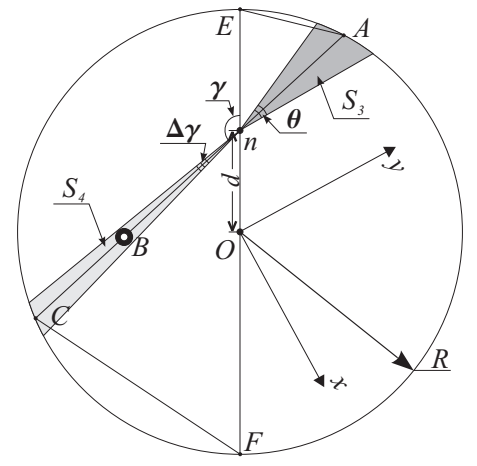

Fig. 5. Calculation of load distribution with a mobile base station.

node $n$ is charged with the (geographical average) forwarding load from a small sector $S_{3}$, when the base station stays at $B$ on segment $n C ; A$ and $C$ are intersections of line $n B$ and circle $\mathcal{C}_{O R}$, and $S_{3}$ is centered around line $n A$ with an angle of $\theta$. This model is not physically as explainable as the model in Section III-A. However, in Appendix II, we show that they are equivalent (in a sense that $\left(S_{1}+S_{2}\right) / S_{2}=S_{3} \rho$ ) and $\theta$ is a decreasing function of $|n B|$. For simplification, we apply an average value $\bar{\theta}=0.2$ that is estimated in Appendix II for any positions of $B$ and $n$ within $\mathcal{C}_{O R}$. In order to facilitate further calculation, we also consider that $B$ is located in another sector $S_{4}$ centered around line $n C$, with an angle of $\Delta \gamma$. When $\Delta \gamma \rightarrow 0$, it goes back to the situation where $B$ is on segment $n C$. Since $B$ visits everywhere within $\mathcal{C}_{O R}$, we divide $d_{i s k_{O R}}$ (the area within $\mathcal{C}_{O R}$ ) into disjoint sectors $\left\{S_{4}^{\gamma}\right\}$ such that $\bigcup_{\gamma} S_{4}^{\gamma}=\operatorname{dis}_{O R}$. Now we calculate the average load of node $n$ by further taking a time average:

$$
\begin{aligned}
\overline{\operatorname{load}}_{n} & =\left.\sum_{\gamma=0}^{2 \pi} \overline{\operatorname{load}}_{n}\right|_{\left\{B \text { in } S_{4}^{\gamma}\right\}} \times \operatorname{Fr}\left\{B \text { in } S_{4}^{\gamma}\right\} \\
& =\sum_{\gamma=0}^{2 \pi} S_{3}^{\gamma} \rho \lambda \varepsilon \times \frac{S_{4}^{\gamma}}{\pi R^{2}} \\
& \approx \sum_{\gamma=0}^{2 \pi} \frac{1}{2}|n A|^{2} \bar{\theta} \rho \lambda \varepsilon \times \frac{\frac{1}{2}|n C|^{2} \Delta \gamma}{\pi R^{2}} \\
& =\sum_{\gamma=0}^{2 \pi} \frac{\left(R^{2}-d^{2}\right)^{2} \bar{\theta} \rho \lambda \varepsilon \Delta \gamma}{4 \pi R^{2}}
\end{aligned}
$$

where $\gamma$ takes only a discrete value of $k \Delta \gamma, k \in \mathbb{Z}^{+}$. The calculation of the occupying frequency $\operatorname{Fr}\left\{B\right.$ in $\left.S_{4}^{\gamma}\right\}$ is based on the assumption that the base station visits everywhere with the same frequency. The reason $|n A|^{2} \times|n C|^{2}=\left(R^{2}-d^{2}\right)^{2}$ is that triangles $\triangle n A E$ and $\triangle n F C$ are similar. Let $\Delta \gamma \rightarrow 0$, the sum over $[0,2 \pi]$ in (3) becomes an integral over $[0,2 \pi]$ :

$$
\begin{aligned}
\overline{\text { load }}_{n} & =\int_{0}^{2 \pi} \frac{\left(R^{2}-d^{2}\right)^{2} \bar{\theta} \rho \lambda \varepsilon}{4 \pi R^{2}} \mathrm{~d} \gamma \\
& =\frac{1}{2}\left(\frac{R^{2}-d^{2}}{R}\right)^{2} \bar{\theta} \rho \lambda \varepsilon
\end{aligned}
$$

The average load is plotted in Fig. 6. A comparison between
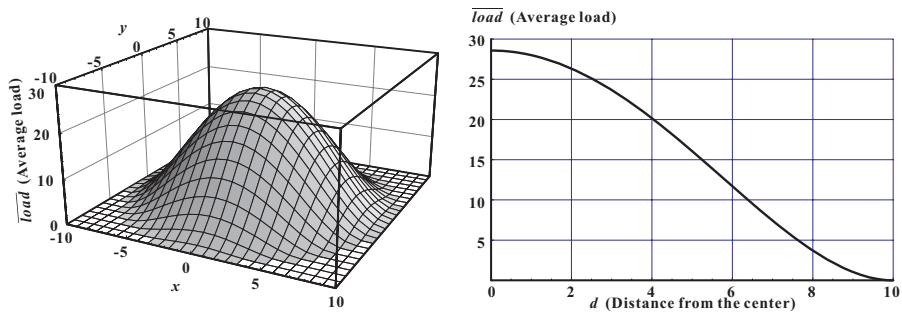

Fig. 6. Load distribution with a mobile base station. We assume $R=10$, $r=1, \bar{\theta}=0.2, \rho=8 / \pi, \lambda=1$ and $\varepsilon=1$.

Fig. 6 and Fig. 4 shows that the maximum average load is much lower in the case of a mobile base station, indicating an extended network lifetime (more than 3 times). We also provide simulation results for these two cases in Section V-A, which corroborate our analysis. In addition, the closed form expression (4) actually suggests that, given certain values for $\lambda$ and $\varepsilon$, there are two possibilities for further improvements:

- Reducing $\theta$ for the hot spot (the center),

- Reducing the network size characterized by $R$.

Note that reducing node density $\rho$ does not help, because, according to Appendix II, $\theta$ increases when decreasing $\rho$.

\section{JOInt MOBILITy AND Routing StRATEgy}

In the previous section, we have shown that mobility helps to balance the load and prolong the network lifetime. This suggests that mobility is indeed a promising strategy to optimize the network lifetime. Now, we refine our problem definition of maximizing network lifetime as:

$$
\begin{array}{cc}
\text { Minimize } & \operatorname{load}_{N} \equiv \max _{\forall n \in N} \overline{\operatorname{load}}_{n}(\mathcal{M}, \mathcal{R}) \\
\text { Constraints : } & \mathcal{M} \text { constraints; } \mathcal{R} \text { constraints }
\end{array}
$$

where $\mathcal{M}$ and $\mathcal{R}$ refer to mobility strategies and routing strategies, respectively. A traditional way of solving the problem with only routing taken into account is linear programming [1], in which the routing constraints are actually flow conservation. However, since we add the mobility strategy, the size of the strategy space increases dramatically. Therefore, we only rely on the following heuristics to achieve a "better" (but not necessarily the "best") solution to the problem: we first fix the routing strategy to short path routing and search for the optimum mobility strategy, then based on the optimum mobility strategy, we search for a routing strategy that performs better than short path routing. The constraints are discussed separately for each strategy. We also explain the implementation issues at the end of this section. 


\section{A. Optimum Mobility Strategy}

We first fix the routing strategy and search for the optimum mobility strategy under the constraint that the base station should not move out of the network region (otherwise it cannot collect data any more). At first glance, the strategy space of mobility is enormous because the number of trajectories that can be chosen is infinite. Fortunately, by defining periodic mobility as recurrent movements with a constant period, we can first reduce the size of the strategy space by removing all aperiodic mobility strategies (i.e., mobility strategies that are not periodic within the network lifetime). In fact, this category of mobility strategies can always be considered as a periodic mobility whose period is the same as the network lifetime. In addition, the following claim further limits our searching to periodic mobility strategies whose trajectories have rotation symmetry of all degrees around the network center (symmetric strategies hereafter for brevity and non-symmetric strategies otherwise):

Claim 2: For each non-symmetric strategy that achieves a network load $\operatorname{load}_{N}$, there exists one corresponding symmetric strategy that achieves a network load no larger than $\operatorname{load}_{N}$.

Proof: Let $\mathcal{T}_{0}$ in Fig. 7(a) be the trajectory of an arbitrary non-symmetric mobility strategy, and let $\operatorname{load}_{N}$ be the network load achieved with $\mathcal{T}_{0}$. In order to obtain the symmetric strat-

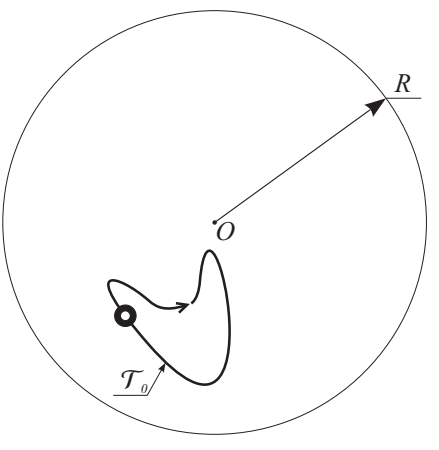

(a) Non-symmetric strategy

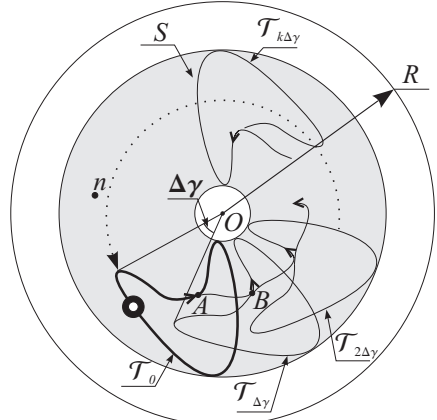

(b) Symmetric strategy
Fig. 7. Transformation from a non-symmetric mobility strategy to a symmetric one.

egy corresponding to $\mathcal{T}_{0}$, we modify the trajectory in such a way that, after completing one period, the base station changes to another trajectory that rotates the previous one about the center with a $\Delta \gamma$ angle. For example, upon completing $\mathcal{T}_{0}$, the base station moves from point $A$ to point $B$ and then starts to move along $\mathcal{T}_{\Delta \gamma}$, as shown in Fig. 7(b). When $\Delta \gamma \rightarrow 0$, the mobility strategy becomes an identical frequency movement ${ }^{3}$ in the annulus $S$ (i.e., the base station appears everywhere in $S$ with the same frequency), which is a symmetric strategy. Note that the mobility strategy we considered in Section III-B is a special case of this category of strategies. Now, let us consider

\footnotetext{
${ }^{3}$ This movement does not necessarily follow the trajectory we suggest here or a random walk (they both have an infinite period); it can always be achieved by a regular moving trajectory (e.g., a space-filling curve [26]) with a short period.
}

the average load of an arbitrary node $n$ in the network. Let $\overline{\text { load }}_{n_{k \Delta \gamma}}$ be the average load of $n$ when only the strategy $\mathcal{T}_{k \Delta \gamma}$ is considered, we have $\overline{l o a d}_{n}=M^{-1} \sum_{k=0}^{k=M} \overline{l o a d}_{n_{k \Delta \gamma}}$, where $M=2 \pi / \Delta \gamma$. Let $\Delta \gamma \rightarrow 0$, we have $\overline{\text { load }}_{n}=$ $(2 \pi)^{-1} \int_{0}^{2 \pi} \overline{l o a d}_{n_{\gamma}} \mathrm{d} \gamma \leq(2 \pi)^{-1} \int_{0}^{2 \pi} \operatorname{load}_{N} \mathrm{~d} \gamma=\operatorname{load}_{N}$, since $\operatorname{load}_{N}$ remains constant with rotation because of the embedded symmetry. Therefore, the network load achieved by the symmetric strategy $\left(\max _{\forall n \in N} \overline{l o a d}_{n}\right)$ is no larger than $\operatorname{load}_{N}$.

This claim actually limits our search to two categories of mobility trajectories: movements on concentric circles and identical frequency movements in annuli, because they are the only symmetric strategies we can have within the network region. Finally, the following claim gives us the best mobility strategy we can have, under the condition of short path routing:

Claim 3: The optimum symmetric strategy is the one whose trajectory is circle $\mathcal{C}_{O R}$ (i.e., the periphery of the network).

A formal proof is hard to achieve in this case, so we instead confirm the claim by analytically comparing mobility trajectories on concentric circles of radius $R_{m}$. With a circle trajectory, we cannot obtain a closed form expression, even with the simplified model of Section III-B. As shown in Fig. 8, we turn back to use the model applied in Section III-A. Similar to (2), we can calculate the average load as follows:

$$
\overline{\operatorname{load}}_{n}=\int_{\gamma} \frac{\left(S_{1}+S_{2}\right) \rho \lambda \varepsilon}{S_{2} \rho} \times \operatorname{Fr}\left\{B \text { on arc } l_{2} \mathrm{~d} \gamma\right\}
$$

where $S_{1} \approx \frac{\beta}{2}\left(\left(l_{1}+l_{2}\right)^{2}-l_{2}^{2}\right)$ and $S_{2} \approx \pi r^{2} / 2$. While $l_{1}=$ $\sqrt{R^{2}-d^{2} \sin ^{2}(\gamma)}+d \cos (\gamma)$, the calculation of $l_{2}$ depends on $d=|n O|$. There are four cases that should be distinguished:

i) $0 \leq d<R_{m}-r \quad$ in Fig. 8(a),

ii) $R_{m}-r \leq d<R_{m} \quad$ in Fig. 8(b),

iii) $R_{m} \leq d<R_{m}+r \quad$ in Fig. 8(c),

iv) $R_{m}+r \leq d<R \quad$ in Fig. 8(d).

In the case of (i), $l_{2}=\sqrt{R_{m}^{2}-d^{2} \sin ^{2}(\gamma)}-d \cos (\gamma)$. Possible situations where $|n B|<r$ have to be taken into account when $R_{m}-r \leq d<R_{m}$. The frequency of such events is $\eta / \pi$, and the average load in that case can be calculated by (2) with $d<r$; otherwise the integral is over $\left[\gamma_{0}, 2 \pi-\gamma_{0}\right]$. The cases of (iii) and (iv) are different because $\gamma$ only varies within $\left[\gamma_{1}, 2 \pi-\gamma_{1}\right]$ and the integration has two parts: a clockwise one and a counter-clockwise one, as shown in Fig. 8(d). The calculation of the counter-clockwise integration is the same as (i). For the clockwise part, $l_{2}=-\sqrt{R_{m}^{2}-d^{2} \sin ^{2}(\gamma)}-$ $d \cos (\gamma)$ and, in the case of (iii), possible situations where $|n B|<r$ are treated in the same way as for (ii).

Assuming $R=10, r=1, \rho=8 / \pi, \lambda=1$, and $\varepsilon=1$, we compute $\overline{\operatorname{load}}_{n}$ with a numerical method. The results for $R_{m}=3,5,7,10$ are plotted in Fig. 9, and the result in Fig. 6 is also re-plotted (denoted by $R_{m} \in[0,10]$ ). It is easy to see that (i) the trajectory with $R_{m}=R$ is the best among all circle trajectories and (ii) the maximum average load is always achieved at the network center. According to these observations and considering the fact that moving 


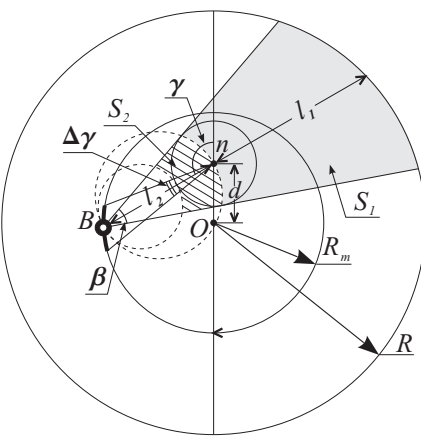

(a) $0 \leq d<R_{m}-r$

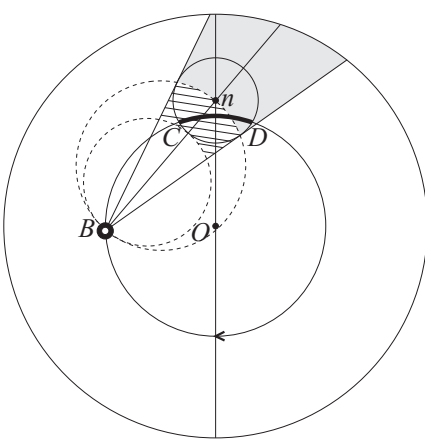

(c) $R_{m} \leq d<R_{m}+r$

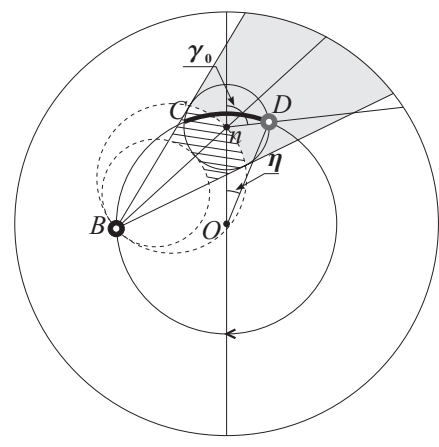

(b) $R_{m}-r \leq d<R_{m}$

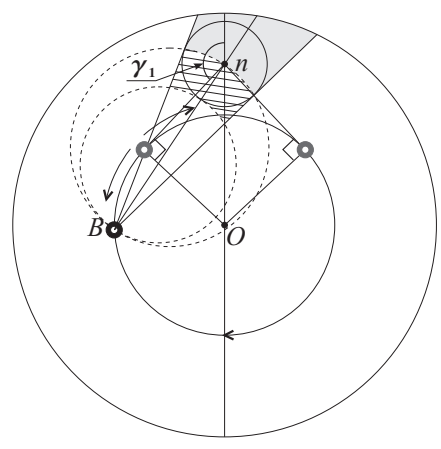

(d) $R_{m}+r \leq d<R$
Fig. 8. Calculation of load distribution with the mobility strategy on a concentric circle.

in an annulus is a weighted average over movements on a set of concentric circles (e.g., the movement with $R_{m} \in$ $[0,10]$ is roughly equivalent to a trajectory of $R_{m}=6$ ), we know that circle $\mathcal{C}_{O R}$ is the optimum mobility strategy under the condition of short path routing. These results prove the correctness of Claim 3. They also match the intuition we get from (4); the trajectory with $R_{m}=R$ maximizes the distance from the base station to the network center (which is always the hot spot), and thus minimizes the angle $\theta$ for the center, which in turn minimizes $\operatorname{load}_{N}$. We validate this analysis by simulations in Section V-B.

\section{B. “Better" Routing Strategy}

According to (4) in Section III-B, there are two ways to reduce the network load. In the previous section, we have already applied one of them (i.e., reducing $\theta$ ) to achieve the optimum mobility strategy. So the only way to further reduce the network load is to decrease $R$. This implies a sacrifice of the network size, if only mobility strategy is considered. Fortunately, we still have another dimension of design strategy, i.e., routing. By investigating the load distribution of networks with a mobile base station (e.g., Fig. 9), we find that the nodes that are near to the border of the network always take a lighter load than the nodes near the center. A "better" routing strategy should exploit the energy capacity of these nodes to compensate the energy consumption of the hot spots. Our heuristic on joint routing and mobility strategy is shown in

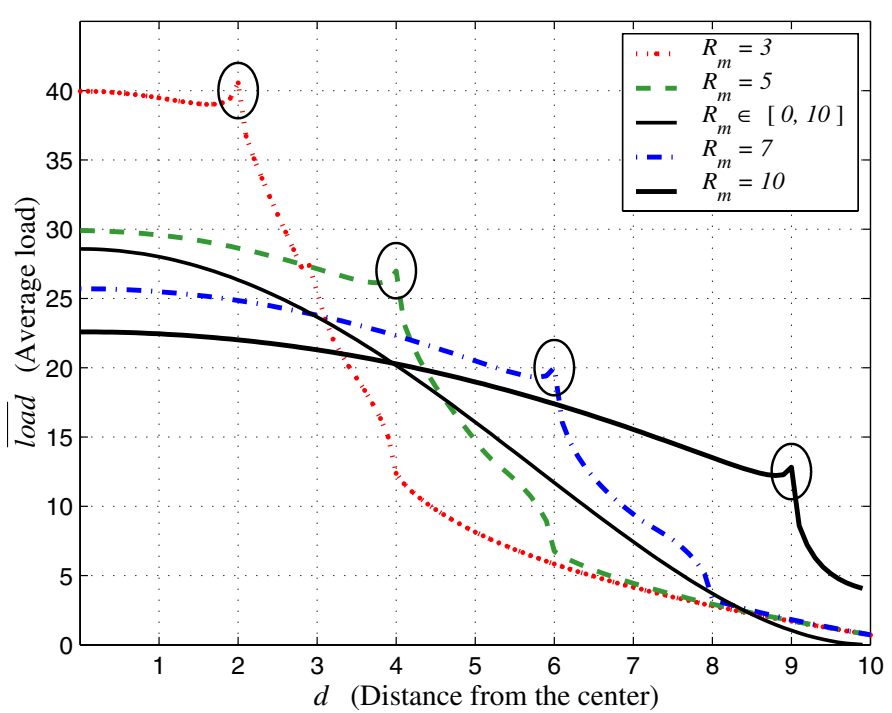

Fig. 9. Comparison between mobility trajectories with different $R_{m}$. The singular regions (circled in the figure) are due to the imperfections of our approximations.

Fig. 10. The network is divided into two parts: an area within

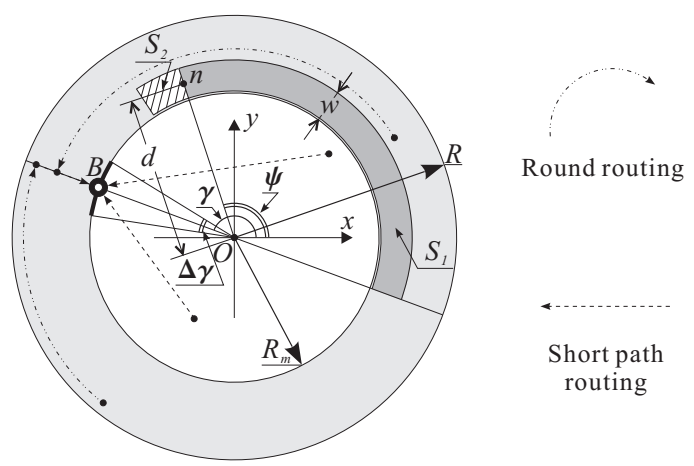

Fig. 10. Joint mobility and routing strategy.

a concentric circle of radius $R_{m}<R$ and an area between that circle and the periphery of the network (the grey annulus). The base station only moves on the circle of radius $R_{m}$. The routing constraints ${ }^{4}$ are such that the nodes within the inner circle still take the short routing path when transmitting data, whereas nodes in the annulus perform a two-step routing: the path first circles around the center $O$ until it reaches $O B$ (round routing hereafter), then it follows a short path to the base station. The direction of the round routing depends on the location of a node: clockwise on one side of the diameter $O B$ and counterclockwise on the other side. The rationale behind this heuristic is that this joint strategy tends to achieve a better performance (i.e., a lower network load) by reducing the radius of the network section that applies short path routing (but not of the whole network) from $R$ to $R_{m}$.

\footnotetext{
${ }^{4}$ Detailed constraints such as flow conservation could be applied for further optimization, but we use only this "high level" specification, because the paper aims at demonstrating the benefit of mobility.
} 
The same analysis in Section IV-A can apply for nodes located within the trajectory circle. Since the radius of this part has been reduced from $R$ to $R_{m}$, the maximum load of this part is approximately $\left(R_{m} / R\right)^{2}$ of the $\operatorname{load}_{N}$ achieved by the optimum mobility strategy (i.e., $R_{m}=R$ ), according to (4). For nodes outside the trajectory circle, a different analysis should be made because those nodes do not apply short path routing. If we could characterize the load distribution for this part of the network, we would choose $R_{m}$ in order to balance the load between these two network sections.

It is easy to model the load distribution if we omit the load incurred by the second routing step. The $S_{1}$ and $S_{2}$ for a node $n$ in this case are shown in Fig. 10. They have the same (radial) width $w$ that is centered on $n$. The length of $S_{1}$ is $d|\psi+\pi-\gamma|$, and the length of $S_{2}$ is always $r$. Note that we are using rectangles to approximate the areas of $S_{1}$ and $S_{2}$, because $w$ can be arbitrarily small. We can then estimate the average load of $n$ with respect to the round routing as follows:

$$
\begin{aligned}
\overline{\text { load }}_{n} & =\int_{\gamma=0}^{2 \pi} \frac{\left(S_{1}+S_{2}\right) \lambda \varepsilon}{S_{2}} \times \operatorname{Fr}\left\{B \text { on arc } R_{m} \mathrm{~d} \gamma\right\} \\
& \approx \int_{0}^{2 \pi}\left(\frac{w d|\psi+\pi-\gamma| \lambda \varepsilon}{r w}+\lambda \varepsilon\right) \times \frac{R_{m} \mathrm{~d} \gamma}{2 \pi R_{m}} \\
& =\left(\frac{d \lambda \varepsilon}{\pi r} \int_{\psi}^{\pi+\psi}(\psi+\pi-\gamma) \mathrm{d} \gamma\right)+\lambda \varepsilon \\
& =\frac{(\pi d+2 r) \lambda \varepsilon}{2 r}
\end{aligned}
$$

However, this model works only for $d-R_{m}>r$. As we will see in Section V-C, the load distribution in the annulus $\left[R_{m}-r, R_{m}+r\right]$ is hard to characterize, simply because, in reality, there is no clear demarcation between the two areas that apply different routing strategies. As a result, we have to rely on simulations to determine the radius $R_{m}$ of a trajectory circle that performs better than the optimum mobility strategy. Nevertheless, the analytical model still provides instructive information. We can compute by (7) that the load taken by nodes near the network periphery is about 17 , given the assumption that $R=10, r=1, \rho=8 / \pi, \lambda=1$, and $\varepsilon=1$. We also know that the maximum load of the inner network section is approximately $\left(R_{m} / R\right)^{2}$ of the $\operatorname{load}_{N}$ achieved by the optimum mobility strategy (which is about 23 from Fig. 9). Therefore, $R_{m}$ should be within [8,10]: smaller values of $R_{m}$ would not lead to lower $\operatorname{load}_{N}$, because $\operatorname{load}_{N} \geq 17$.

\section{Implementation Issues}

We now explain the implementation aspects of the aforementioned mobility and routing strategies. The explanations are expressed as the answers to four questions.

1) How to make a base station move? We can mount a sensor node (e.g., a Berkeley mote [27]) on a robot. The robot provides a power supply for the node and also carries communication facilities that enable the base station to communicate with devices outside the considered sensor network. A detailed design of a mobile base station is out of the scope of this paper; a brief introduction can be found in [13], [28]. It is important to note that, in order to achieve a balanced load distribution, the mobility period (i.e., the time to complete one round along a trajectory) should divide the network lifetime. This implies, in practice, a period much shorter than the lifetime.

2) How to achieve short path routing in the case of a mobile base station? We need to consider two cases:

a) The base station performs a discrete movement whose trajectory coincides with sensor locations: The routing is easy to achieve in this case, if there is a (loose) time synchronization among nodes. The base station itinerary (a discrete trajectory along with a time schedule) and the pairwise routing path can be determined after the deployment but before the operation of the network. As a result, each node knows the location of the base station at a given time and which routing path it should use to transmit data. Since the routing computation is performed offline, we can apply any sophisticated loadbalanced routing protocol for all nodes.

b) The base station performs a continuous movement that follows the exact curve of a trajectory: The base station has to broadcast a data query whenever the network topology changes due to the mobility, in order to refresh the routing information of the nodes. This scheme does not necessarily bring extra overhead, because sensor networks with a static base station also need periodic query flooding [17] and because practical routing protocols (e.g., MintRoute [29]) apply a table-driven scheme. If the base station and the sensor nodes are locationaware (using GPS or other localization methods [30], [31], [32]) and the base station announces its location to all nodes, building load-balanced routing path online is possible [4].

3) How to implement the round routing? The trajectory based forwarding, proposed in [33], [34], provides a way to shape a routing path into a predefined curve. In our case, the curve is simply an arc parameterized by a radius and the coordination of its center. The location-awareness is necessary for sensor nodes that apply such a routing, which brings extra overhead and could potentially offset the benefit of our joint strategy. Therefore, a careful design based on field testing is needed.

4) What if the network region is not circular? The periphery mobility strategy can be conjectured as being (at least) nearly optimum, because it could be the best way to disperse the traffic flows. Note that this strategy also has a practical significance: certain applications (e.g., habitat monitoring [35]) of sensor networks do prefer less human intervention in the inner part of the networks in order to reduce the disturbance effects. A joint strategy depends heavily on the shape of the network region, but the idea of exploiting redundant energy capacity always applies.

\section{Simulations}

In this section, we provide simulation results for the strategies presented in Section III and IV, including the static base station, the mobile base station, and the joint mobility and routing. We also compare these results with their corresponding analytical results. We perform simulations with a high 


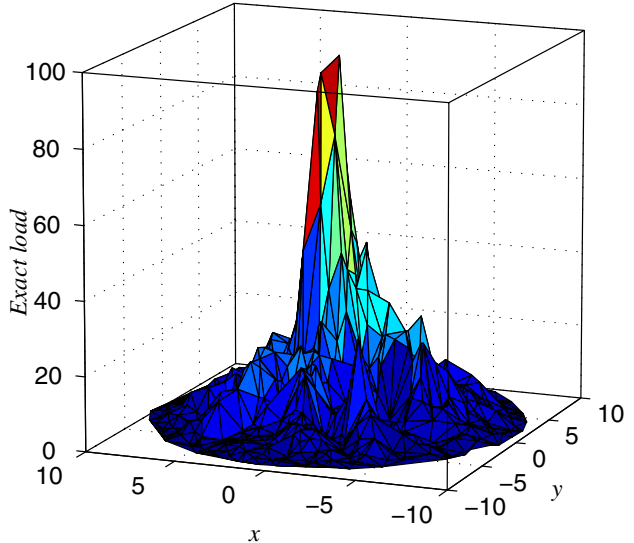

(a)

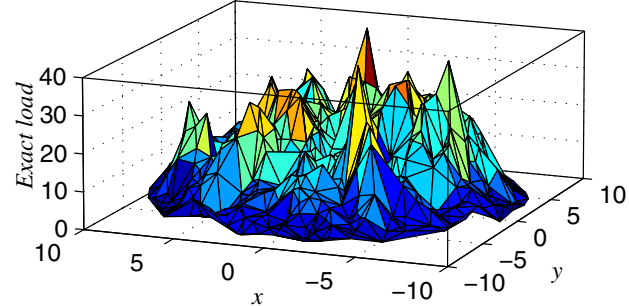

(b)

Fig. 11. Illustration of the exact load distribution: (a) static base station and (b) mobile base station.

level simulator programmed in MATLAB ${ }^{\circledR}$, which ignores the MAC effects.

Our simulation set-ups consist of about 800 nodes deployed within a circle of $R=10$ units. The nodes are randomly scattered as a Poisson process with density $\rho=8 / \pi$. Each node has a transmission range of $r=1$ unit. We also normalize $\lambda$ (data rate), $\varepsilon$ (energy consumption for a data unit), and $T$ (simulation time) to 1 . For strategies with a mobile base station, we always consider a discrete (in the sense of both space and time) mobility trajectory for the base station. So if a mobility trajectory consists of $m$ steps, the base station spends $1 / m$ time for each step. We also assume that the base station only stays at sensor locations in order to keep the network connectivity independent of the positions of the base station; this results in an actual mobility trajectory that does not exactly follow the defined trajectory (e.g., a circle). We emulate the effect of load-balanced routing by randomizing all link weights before searching a routing path; this scheme distributes the traffic forwarding load from other nodes among a set of neighboring nodes. For each strategy, we perform 10 simulations with different node deployments.

\section{A. Static vs. Mobile}

We perform simulations for the two strategies analyzed in Section III. A static base station is located at the node whose distance to the network center is the smallest, and a mobile base station stays at each node for a time period of $1 /|N|$ (remember that we have $|N| \approx 800$ nodes in a network).

Fig. 12 compares the simulation results with the analytical ones. The figure represents the average load of nodes versus their distance $d$ from the center. The analytical results are simply a re-plot of the curves in Fig. 4 and 6. Simulation results match the analytical ones very well in the case of a static base station. For the mobile base station strategy, analytical results appear to be a bit over-optimistic, but they do not differ too much from the simulation results when $d$ varies between 0 and 5 . The difference becomes larger when $d$ goes beyond 5; the reason is that we apply the $\bar{\theta}$ estimation

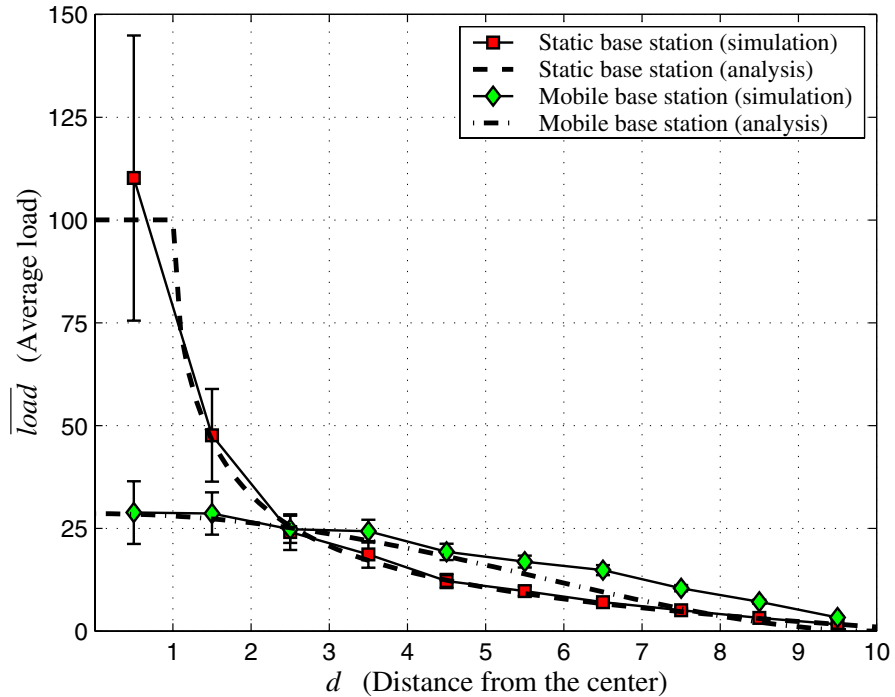

Fig. 12. Comparison between analytical and simulation results on the average load (confidence intervals $95 \%$ ).

of the center for every other nodes. In spite of these errors, the overall approximation is valid. Most importantly, it is observed that a mobile base station reduces $\operatorname{load}_{N}\left(\equiv \max _{\forall n \in N} \overline{\operatorname{load}}_{n}\right)$ by about $75 \%$; this implies a $400 \%$ increase of the network lifetime.

We also evaluate the network lifetime defined as the time for the first node to die [1], [36], which is often pessimistic [22]. Fig. 11 shows the simulation results of the exact (not average) load distribution for one of the network configurations. One can easily see the similarity between these plots and Fig. 4 and 6. The unusual spikes appearing in Fig. 11(b) (compared with Fig. 6) are due to three facts: (i) the network topology is not regular, (ii) the movement of the base station is not continuous, and (iii) the emulation of load-balanced routing is not perfect. However, the gain in lifetime is still quite obvious even with these spikes. Since the maximum load taken by a 
node is reduced by about $60 \%$, the lifetime is increased by about $250 \%$.

\section{B. Optimum Mobility with Short Path Routing}

We evaluate different mobility strategies (under the condition of short path routing) in this section. We make a comparison between three strategies: (i) movement on a concentric circle of radius $R_{m}=5$, (ii) identical frequency movement in the network (the one described in Section III-B, equivalent to $R_{m} \in[0,10]$ ), and (iii) peripheral movement with $R_{m}=10$. Each discrete movement on a circle consists of 72 steps, so a mobile base station stays at each step for a time period of 1/72. Fig. 13 shows the comparison results. It actually confirms our Claim 3 that peripheral movement is the best strategy. Furthermore, the comparison between analytical and simulation results also prove the validity of our models. Finally, we observe that the identical frequency movement is better than the circle movement of $R_{m}=5$ and is worse than the peripheral movement. This result supports our argument that the identical frequency movement is a weighted average over all the circle movements it covers.
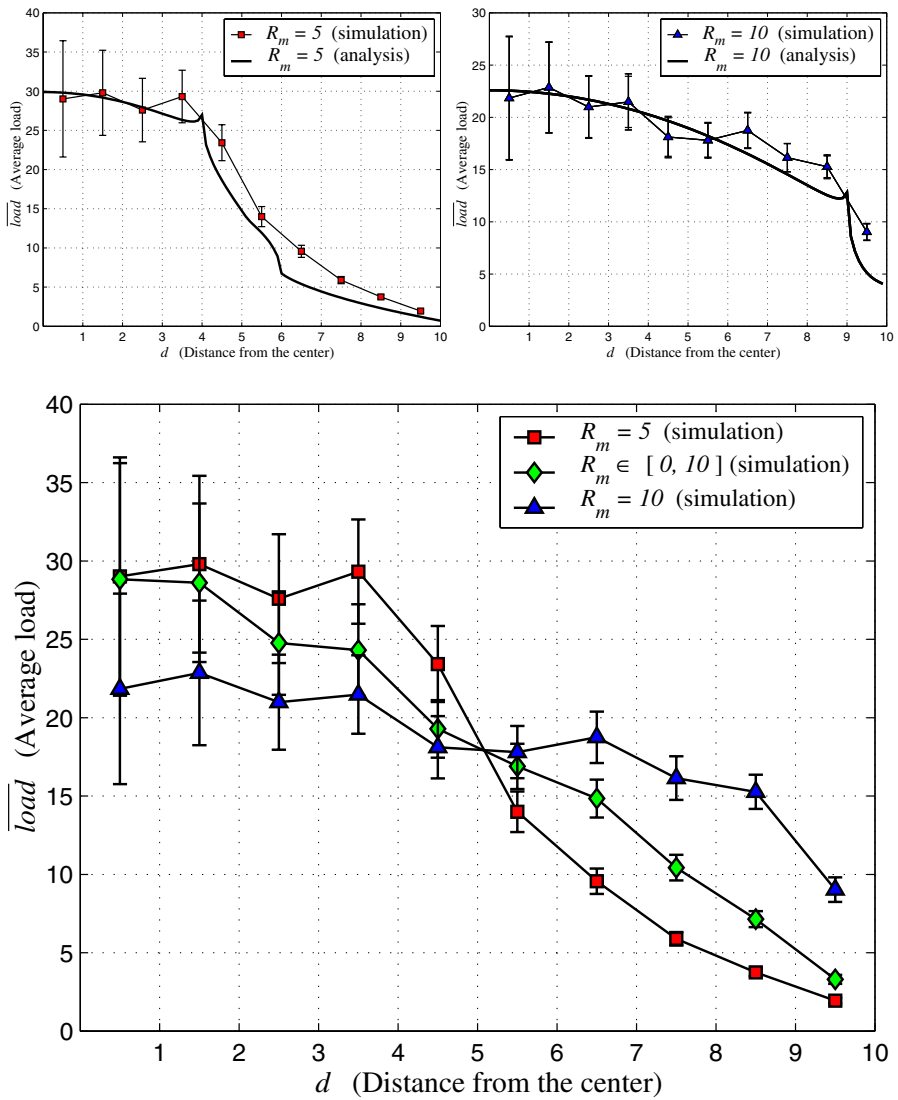

Fig. 13. Comparison between different mobility strategies (confidence intervals $95 \%$ ).

\section{Joint Mobility and Routing}

In this section, we provide simulation results for the joint strategy proposed in Section IV-B. The results plotted in
Fig. 14 show that the joint strategy indeed reduces the maximum load in the network section within the mobility trajectory, at the cost of an increased load in the network section outside the mobility trajectory. The maximum load of the two network sections becomes equal when $R_{m}=9$, which gives us the best choice of the joint strategy. The joint strategy further reduces the network load by about $10 \%$ compared with the optimum mobility strategy $\left(R_{m}=10\right)$. As a result, the overall improvement of the network lifetime, compared with the case of static base station (Fig. 12), reaches about $500 \%$.

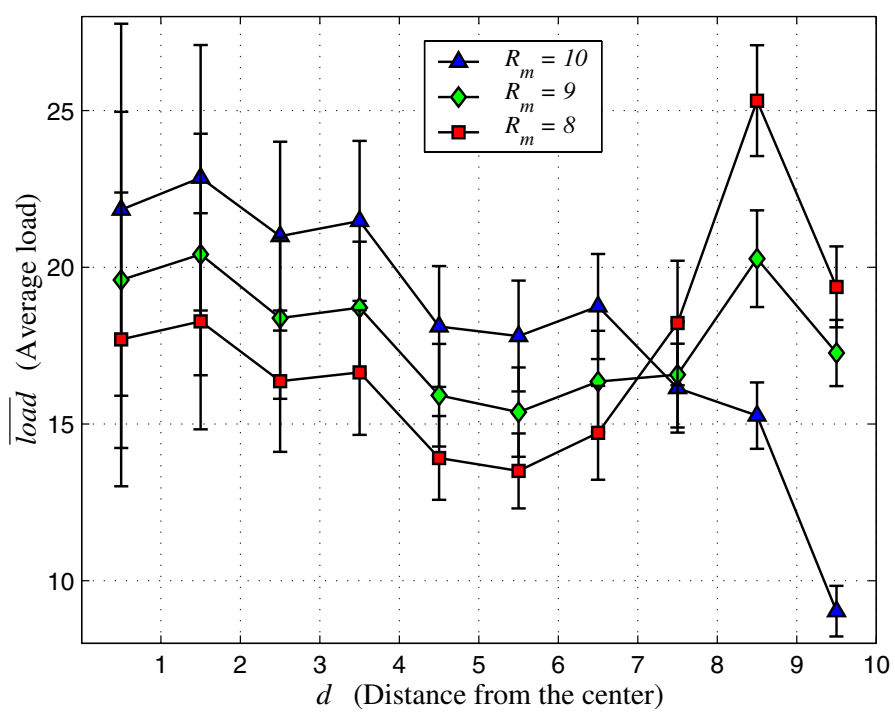

Fig. 14. Comparison between different joint mobility and routing strategies (confidence intervals 95\%).

\section{RELATED WORK}

The existing work concerned with lifetime (or energy conservation) issues is so vast that it would deserve several comprehensive surveys. Hence, we only discuss a few related topics here. Energy conserving routing (e.g., [1], [2], [3], [4]) aims at balancing the energy consumption among all nodes, instead of minimizing the absolute consumed power. Topology control (e.g., [5], [6], [7], [8]) and clustering (e.g., [9], [10], [11], [12]) are usually closely correlated [11]. They both manipulate the network topology by exploiting the nodes' ability to adjust their transmission power dynamically; the goal is to maintain network connectivity while reducing energy consumption and improving network capacity. Although these proposals are somewhat orthogonal to the idea we present in this paper, they are all potentially complementary to our idea.

Base station mobility has also been exploited to extend the network lifetime [15], [16], but in a form of mobile relay approach (an idea originally described in [37]), which can lead to significant delays of data delivery. Also, their proposals leverage only on uncontrollable (although predictable for [16]) mobility of the base station. The controllable mobility was recently investigated in [28]. This proposal is a compromise between the mobile relay approach and ours: the base station 
still relays data with its movements, but a node that does not lie in the vicinity of the mobility trajectory transmits data through a multi-hop routing when the base station moves to the closest point to the node. It would be interesting to have a theoretical analysis on this hybrid approach. Our proposal differs from [15], [16], [28] in that we do not apply the mobile relay approach; as a result, the moving speed, which is crucial in their cases due to its impact on latency, is not essential any more to our solution. Finally, it is also important to note that the mobility of network nodes can facilitate sensor deployment [38], reduce sensing uncertainty [39], and act as a network control primitive to improve communication performance in ad hoc networks [40].

In terms of the model we have used, two papers should be mentioned. Pham and Perreau [41] propose a similar model to analyze the performance of multi-path routing. Ganjali and Keshavarzian [25] introduce another model for the same purpose.

\section{CONCLUSION}

In this paper, we have first shown that, with a static base station, the sensor nodes located close to the base station suffer premature battery depletion, leading to an early disconnection of the network. In order to better balance the load among the nodes, we have proposed that the base station be mobile and demonstrated that the traffic experienced by the most heavily loaded nodes is reduced by a factor of 3 with only an arbitrary mobility strategy. These results suggest that base station mobility is indeed a strategy that deserves to be considered when optimizing the network lifetime. In order to further exhibit the benefit of the base station mobility, we have compared different mobility strategies and obtained the optimum one under the assumption of certain routing strategies. We have also explained how routing can be fine-tuned to leverage on the trajectory of the base station; in particular, we have shown how to better exploit the transmission capabilities of the nodes located at the periphery of the network. Our joint mobility and routing strategy achieves a $500 \%$ improvement of the network lifetime. For all these scenarios, we have developed an analytical model, corroborated by a set of simulations.

We are currently considering an interesting complement to the work in this paper, where we perform an analysis based on a discrete model (i.e., a graph). In addition, we are preparing field tests that could better justify the benefit of the base station mobility.

In terms of other future work, we intend to study how data aggregation protocols can be efficiently integrated into the proposed scheme. We will also explore how a set of mobile base stations can be coordinated to further optimize the data collection.

More generally, an intriguing research area would consist in further exploiting the capabilities ${ }^{5}$ of base stations for optimizing various aspects of sensor networks.

\footnotetext{
${ }^{5}$ As shown in [14], in addition to mobility, a robot can also calibrate and recharge sensor nodes.
}

\section{ACKNOWLEDGEMENTS}

The authors would like to a give special thank to Mark Felegyhazi who helped to initiate this research. The authors would also like to thank Olivier Dousse, Razvan Cristescu, Imad Aad, Jacques Panchard, Henri Dubois-Ferriere, and Michal Piorkowski for several instructive discussions. Finally, the authors wish to thank the anonymous reviewers for their valuable feedback.

\section{APPENDIX I \\ Modeling the Load of Sensor Nodes: The Case of a STATIC BASE STATION}

We sketch the analytical model proposed by Ganjali and Keshavarzian [25] and show how we extend their model for our analysis. Let us consider two fixed points $B$ and $n$ (which refer to the base station and an arbitrary node in our case). Ganjali and Keshavarzian observe that the set of nodes used by routing paths between any two nodes approximately lie in a rectangle of width $2 w$. They suggest that the locus of a node $x$ whose routing path towards $B$ goes through $n$ should meet two conditions if $|x B| \geq w$ : (i) the distance from $n$ to the line segment $x B$ should be less than $w$, and (ii) the projection of point $n$ on $x B$ should lie between $x$ and $B$. These two criteria indicate that the locus of $x$ is the area $S_{1}$ in Fig. 15(a). In our case, we only assume single-path routing, so it is intuitively correct to set $w=r$.

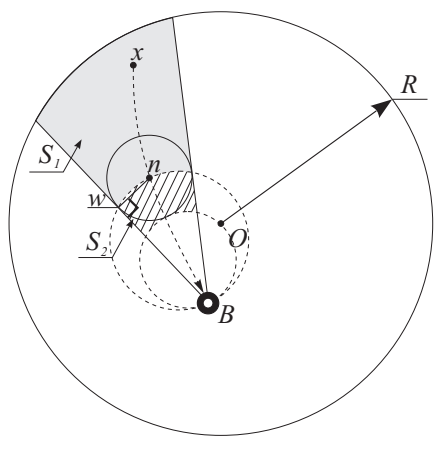

(a) $|n B| \geq w$

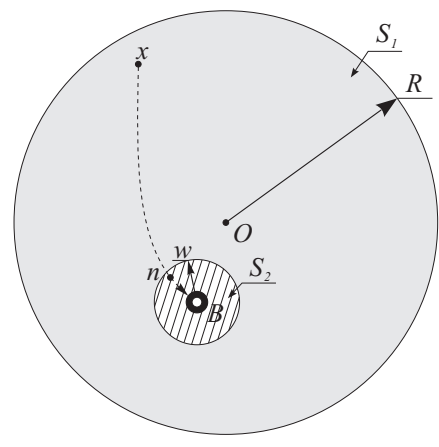

(b) $|n B|<w$
Fig. 15. Modeling sensor load in the case of a static base station.

Actually, it is only in the worst case that $n$ should forward all traffic flows from $S_{1}$. Since we apply an ideal load-balanced routing, the load will be shared among nodes in $S_{2}$, i.e., nodes within this one-hop belt have the same probability to be chosen as forwarding nodes. As a result, $\overline{l o a d}_{n}$ is in proportion to $\left(S_{1}+S_{2}\right) / S_{2}$ (remember that nodes in $S_{2}$ have to forward their own data traffic). Directly calculating the area of $S_{2}$ leads to a very complex expression, but the area can be approximated by $\pi r^{2} / 2$, as suggested in Fig. 15(a). When $|n B|<r$, [25] concludes that the locus of $x$ consists of the intersection of a half plane and the network region. This result, however, does not apply to our average load computation. For average load evaluation, it is easy to see that nodes (including $n$ ) within the transmission range $r$ of $B$ share the load of forwarding traffic 
flows from all nodes in the networks, as shown in Fig. 15(b), again due to our assumption of an ideal load-balanced routing.

\section{APPENDIX II}

Modeling the Load of Sensor Nodes: The Case of A MOBILE BASE STATION

For the case of a mobile base station, it is very hard to obtain a closed form expression for $\overline{l o a d}_{n}$ if we continue using the model in Appendix I. The reason is that the angle $\beta$ in Fig. 16(a), and thus the area of $S_{1}$, depends on the positions of both $n$ and $B$. Let us consider a new model where node $n$ has the duty of forwarding data traffic from $S_{3}$, a sector centered around $n A$ with an angle of $\theta$. This model is equivalent to the model in Appendix I if $\left(S_{1}+S_{2}\right) / S_{2}=S_{3} \rho$. Given a position of $n$ and an angle $\gamma$, since $S_{1}$ decreases with increasing $|n B|$ and $S_{2}$ is somewhat constant, $S_{3}$, and thus $\theta$, is a decreasing function of $|n B|$. If we can find a sector $\overline{S_{3}}$ of angle $\bar{\theta}$ that is equivalent to $\left(S_{1}+S_{2}\right) / S_{2}$ on average but is decoupled from $|n B|$, the estimations provided in Section III-B would be justified. However, obtaining a value equivalent to $\left(S_{1}+\right.$

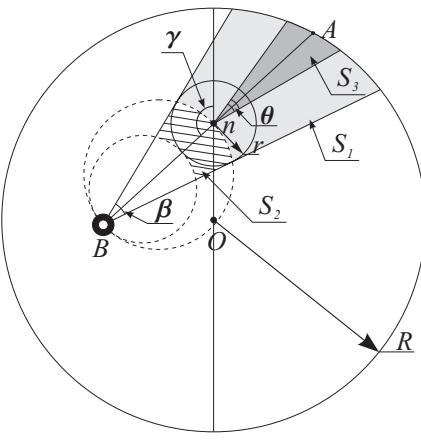

(a) Arbitrary node $n$

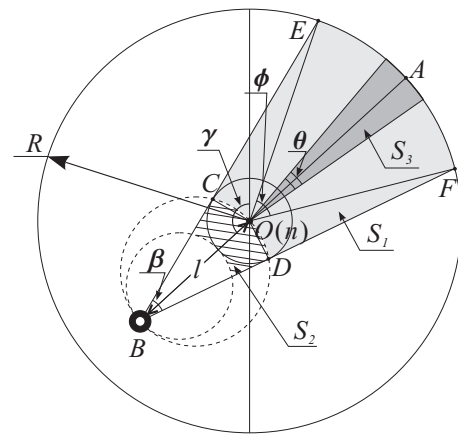

(b) Centered node $n$
Fig. 16. Modeling sensor load in the case of a mobile base station.

$\left.S_{2}\right) / S_{2}$ on average for an arbitrary $n$ has the same complexity as calculating the $\overline{\operatorname{load}}_{n}$. Fortunately, $\bar{\theta}$ is computable when $n$ lies on the center, as shown in Fig. 16(b). Also, it is observed that the area of $S_{3}$ depends mostly on its height, i.e., $|n A|$ in both Fig. 16(a) and (b), instead of $\theta$, since $S_{3} \approx \theta|n A|^{2} / 2$. So we can calculate $\bar{\theta}$ for the centered $n$ and apply this value as an estimation for an arbitrary $n$. Let $\overline{l o a d}_{n}$ be the value computed with $\left(S_{1}+S_{2}\right) / S_{2}$ and $\widehat{l o a d}_{n}$ be the value computed with $\bar{\theta}$ (thus $\bar{S}_{3}$ ), we want $\overline{\operatorname{load}}_{n}=\widehat{\operatorname{load}}_{n}$ for a centered $n$. These two values can be computed as follows:

$$
\begin{aligned}
\widehat{\operatorname{load}}_{n} & =\int_{\gamma=0}^{2 \pi} \int_{l=0}^{R} S_{3} \rho \lambda \varepsilon \times \operatorname{Fr}\{\text { base station at } B\} \\
& =\int_{0}^{2 \pi} \int_{0}^{R} \frac{1}{2} \bar{\theta} R^{2} \rho \lambda \varepsilon \times \frac{l \times \mathrm{d} l \times \mathrm{d} \gamma}{\pi R^{2}} \\
& =\frac{1}{2} \bar{\theta} R^{2} \rho \lambda \varepsilon
\end{aligned}
$$

$$
\begin{aligned}
\overline{\text { load }}_{n} & =\int_{\gamma=0}^{2 \pi} \int_{l=0}^{R} \frac{\left(S_{1}+S_{2}\right) \lambda \varepsilon}{S_{2}} \times \operatorname{Fr}\left\{\text { base station }{ }^{6} \text { at } B\right\} \\
& \approx\left\{\int_{0}^{2 \pi} \int_{r}^{R} \frac{\left(S_{O E F}+S_{O C E}+S_{O D F}+S_{2}\right) \lambda \varepsilon}{S_{2}}\right. \\
& \left.\times \frac{l \times \mathrm{d} l \times \mathrm{d} \gamma}{\pi R^{2}}\right\} \\
& +\int_{0}^{2 \pi} \int_{0}^{r} \frac{\pi R^{2} \lambda \varepsilon}{\pi r^{2}} \times \frac{l \times \mathrm{d} l \times \mathrm{d} \gamma}{\pi R^{2}} \\
& \approx \frac{4 \lambda \varepsilon}{\pi r^{2} R^{2}}\left\{\int _ { r } ^ { R } \left(\left(\arcsin \left(\frac{r}{l}\right)+\arcsin \left(\frac{r}{R}\right)\right) R^{2}\right.\right. \\
& \left.\left.+r \sqrt{R^{2}-r^{2}}+\frac{\pi r^{2}}{2}\right) l \mathrm{~d} l\right\}+\lambda \varepsilon
\end{aligned}
$$

Let $\overline{\text { load }}_{n}=\widehat{\text { load }}_{n}$ and assume $R=10, r=1 \rho=8 / \pi$, $\lambda=1$, and $\varepsilon=1$; we thus have $\bar{\theta} \approx 0.2$.

\section{REFERENCES}

[1] J.-H. Chang and L. Tassiulas, "Energy Conserving Routing in Wireless Ad-hoc Networks," in Proc. of the 19th IEEE INFOCOM, 2000.

[2] K. Kar, M. Kodialam, T.V. Lakshman, and L. Tassiulas, "Routing for Network Capacity Maximization in Energy-constrained Ad-hoc Networks," in Proc. of the 22rd IEEE INFOCOM, 2003.

[3] A. Sankar and Z. Liu, "Maximum Lifetime Routing in Wireless Ad-hoc Networks," in Proc. of the 23rd IEEE INFOCOM, 2004.

[4] J. Gao and L. Zhang, "Load Balanced Short Path Routing in Wireless Networks," in Proc. of the 23rd IEEE INFOCOM, 2004.

[5] L. Li, J.Y. Halpern, P. Bahl, Y.-M. Wang, and R. Wattenhofer, "Analysis of A Cone-based Distributed Topology Control Algorithm for Wireless Multi-hop Networks," in Proc. of the 20th ACM PODC, 2001.

[6] J. Pan, Y.T. Hou, L. Cai, Y. Shi, and S.X. Shen, "Topology Control for Wireless Sensor Networks," in Proc. of the 9th ACM MobiCom, 2003.

[7] N. Li and J. Hou, "Topology Control in Heterogeneous Wireless Networks: Problems and Solutions," in Proc. of the 23rd IEEE INFOCOM, 2004.

[8] X.-Y. Li, Y. Wang, P.-J. Wan, W.-Z. Song, and O. Frieder, "Localized Low-Weight Graph and Its Applications in Wireless Ad Hoc Networks," in Proc. of the 23rd IEEE INFOCOM, 2004.

[9] W.R. Heinzelman, A. Chandrakasan, and H. Balakrishnan, "An Application-specific Protocol Architecture for Wireless Microsensor Networks," IEEE Trans. on Wireless Communications, vol. 1, no. 4, pp. 660-670, 2002.

[10] V. Kawadia and P.R. Kumar, "Power Control and Clustering in Ad Hoc Networks," in Proc. of the 22nd IEEE INFOCOM, 2003.

[11] L. Bao and J.J. Garcia-Luna-Aceves, "Topology Management in Ad Hoc Networks," in Proc. of the 4th ACM MobiHoc, 2003.

[12] O. Younis and S. Fahmy, "Distributed Clustering in Ad-hoc Sensor Networks: A Hybrid, Energy-Efficient Approach," in Proc. of the 23rd IEEE INFOCOM, 2004.

[13] J. Butler, "Robotics and Microelectronics: Mobile Robots as Gateways into Wireless Sensor Networks," Technology@Intel Magazine, May 2003.

[14] A. LaMarca, W. Brunette, D. Koizumi, M. Lease, S. Sigurdsson, K. Sikorski, D. Fox, and G. Borriello, "Making Sensor Networks Practical with Robots," in LNCS, F. Mattern and M. Naghshineh, Eds., pp. 152-166. Springer-Verlag, 2002.

[15] R.C. Shah, S. Roy, S. Jain, and W. Brunette, "Data MULEs: Mobeling a Three-tier Architecutre for Sparse Sensor Networks," in Proc. of the 1st IEEE SNPA, 2003.

[16] A. Chakrabarti, A. Sabharwal, and B. Aazhang, "Using Predictable Observer Mobility for Power Efficient Design of Sensor Networks," in Proc. of the 2nd IEEE IPSN, 2003.

${ }^{6}$ By "base station at $B$ ", though an abuse of terminology, we mean that the base station is in an infinitesimal area (which measures $l \times \mathrm{d} l \times \mathrm{d} \gamma$ in polar coordinates) centered on $B$. 
[17] C. Intanagonwiwat, R. Govindan, D. Estrin, J. Heidemann, and F. Silva, "Directed diffusion for wireless sensor networking," IEEE/ACM Trans. on Networking, vol. 11, no. 1, pp. 2-16, 2003.

[18] D. Petrovic, R.C. Shah, K. Ramchandran, and J. Rabaey, "Data funneling: Routing with aggregation and compression for wirless sensor networks," in Proc. of the 1st IEEE SNPA, 2003.

[19] http://commonsense.epfl.ch/, ,"

[20] M. Bhardwaj and A.P. Chandrakasan, "Bounding the Lifetime of Sensor Networks Via Optimal Role Assignments," in Proc. of the 21st IEEE INFOCOM, 2002.

[21] M. Cagalj, J.-P. Hubaux, and C. Enz, "Minimum-energy Broadcast in All-wireless Networks: NP-completeness and Distribution Issues," in Proc. of the 8th ACM MobiCom, 2002.

[22] D.M. Blough and P. Santi, "Investigating Upper Bounds on Network Lifetime Extenstion for Cell-based Energy Conservation Techniques in Stationary Ad Hoc Networks," in Proc. of the 8th ACM MobiCom, 2002.

[23] H. Zhang and J. Hou, "On Deriving the Upper Bound of $\alpha$-Lifetime for Large Sensor Networks," in Proc. of the 5th ACM MobiHoc, 2004

[24] Z. Hu and B. Li, "On the Fundamental Capacity and Lifetime Limits of Energy-Constrained Wireless Sensor Networks," in Proc. of the 10th IEEE RTAS, 2004.

[25] Y. Ganjali and A. Keshavarzian, "Load Balancing in Ad Hoc Networks: Single-path Routing vs. Multi-path Routing," in Proc. of the 23rd IEEE INFOCOM, 2004

[26] H. Sagan, Space-Filling Curves, Springer-Verlag, New York, 1994.

[27] M. Horton, D. Culler, K. Pister, J. Hill, R. Szewczyk, and A. Woo, "MICA, the commercialization of microsensor motes," Sensors Magazine, vol. 19, no. 4, pp. 40-48, 2002

[28] A. Kansal, A. Somasundara, D.D. Jea, M.B. Srivastava, and D. Estrin, "Intelligent Fluid Infrastructure for Embedded Networks," in Proc. of the 2nd ACM/USENIX MobiSys, 2004.

[29] A. Woo, T. Tong, and D. Culler, "Taming the Underlying Challenges of Reliable Multihop Routing in Sensor Networks," in Proc. of the 1st ACM SenSys, 2003.
[30] S. Capkun, M. Hamdi, and J.-P. Hubaux, "GPS-free Positioning in Mobile Ad-Hoc Networks," Cluster Computing Journal, vol. 5, no. 2, pp. 118-124, 2002.

[31] T. He, C. Huang, B.M. Blum, J.A. Stankovic, and T. Abdelzaher, "Range-free Localization Schemes for Large Scale Sensor Networks," in Proc. of the 9th ACM MobiCom, 2003.

[32] A. Rao, S. Ratnasamy, C. Papadimitriou, S. Shenker, and I. Stoica, "Geographic Routing without Location Information," in Proc. of the 9th ACM MobiCom, 2003.

[33] D. Niculescu and B. Nath, "Trajectory Based Forwarding and It Applications," in Proc. of the 9th ACM MobiCom, 2003.

[34] M. Yuksel, R. Pradhan, and S. Kalyanaraman, "An Implementation Framework for Trajectory-Based Forwarding in Ad-Hoc Networks," in Proc. of IEEE ICC, 2004.

[35] A. Mainwaring, J. Polastre, R. Szewczyk, D. Culler, and J. Anderson, "Wireless Sensor Networks for Habitat Monitoring," in Proc. of the 1st ACM WSNA, 2002.

[36] E.J. Duarte-Melo, M. Liu, and A. Misra, "A Modeling Framework for Computing Lifetime and Information Capacity in Wireless Sensor Networks," in Proc. of the 2nd WiOpt, 2004.

[37] M. Grossglauser and D. Tse, "Mobility increases the capacity of ad hoc wireless networks," IEEE/ACM Trans. on Networking, vol. 10, no. 4, pp. 477-486, 2002.

[38] G. Wang, G. Cao, and T. La Porta, "Movement-Assisted Sensor Deployment," in Proc. of the 23rd IEEE INFOCOM, 2004.

[39] M.A. Batalin, M. Rahimi, Y. Yu, D. Liu, A. Kansal, G.S. Sukhatme, W.J. Kaiser, M.Hansen, G.J. Pottie, M.Srivastava, and D. Estrin, "Call and Response: Experiments in Sampling the Environment," in Proc. of the 2nd ACM SenSys, 2004.

[40] D. Goldenberg, J. Lin, A.S. Morse, B. Rosen, and Y.R. Yang, "Towards Mobility as a Network Control Primitive," in Proc. of the 5th ACM MobiHoc, 2004.

[41] P.P. Pham and S. Perreau, "Performance Analysis of Reactive Shortest Path and Multi-path Routing Mechanism with Load Balance," in Proc. of the 22nd IEEE INFOCOM, 2003. 